\title{
Migración: la construcción social de una experiencia
}

\author{
Graciela Sarrible \\ Universitat de Barcelona \\ Diagonal, 690. 08034 Barcelona \\ sarrible@eco.ub.es
}

\section{Resumen}

Las primeras migraciones de los argentinos hacia Europa, a posteriori del golpe militar, concentraron muchos profesionales y personas que no pudieron optar, que no tuvieron una verdadera o completa elección. A pesar de las circunstancias en que se realizó, muchas de ellas, ya de retorno, valoraron positivamente la etapa al considerar que habían aprendido mucho y que podían aportar sus experiencias en la nueva etapa democrática. Existe diversidad entre los que retornaron y aquellos que permanecen en España. La encuesta cualitativa que recoge estas experiencias fue realizada en Buenos Aires y Barcelona.

Palabras clave: migración internacional, construcción social, argentinos.

\begin{abstract}
In the first international migrations from Argentina to Europe, after the military government arrived, there were many professionals and persons that couldn't chose because a free election was impossible in that moment. Though the context of migration was not free, not advantageous, persons that return to Argentina has made a positive evaluation of the period they were out of the country. They expressed that they have learned a lot and that there experienced can be useful for the society in the new democratic period. There are differences between persons living in Spain and migrants that have returned. This article is based in a qualitative survey made in Buenos Aires and Barcelona.
\end{abstract}

Key words: international migration, social construction, argentines.

\section{Sumario}

Introducción Migrar: una experiencia positiva,

Las negativas a aceptar las entrevistas no siempre recomendable

La construcción de la experiencia La migración forma parte del pasado

Bibliografía 


\section{Introducción}

Mientras que en España la cantidad de extranjeros que reside se incrementa, existen comunidades precisas, cuando se las distingue por origen, que pierden número de efectivos. Esto no significa, exactamente, que el número de personas de ese origen que reside en España, disminuya. Entran y salen en un movimiento permanente, pero también cambian de categoría: obtener la nacionalidad permite a un extranjero seguir residiendo en España, al mismo tiempo que merma el número de las personas que han nacido y tienen esa nacionalidad de origen. Hace tiempo que en las estadísticas y estudios de migraciones en países europeos donde los movimientos son de anterior data o período, se cuentan las nacionalizaciones para poder estimar de un modo más exacto las personas que residen en el territorio y han venido del extranjero.

En el caso de los argentinos en España, el movimiento es continuo y las migraciones han ido cambiando en el tiempo con las motivaciones y los procesos políticos que se viven en el país de origen. Mientras que las de los años setenta y principios de los ochenta tenían, sobre todo, razones políticas, las posteriores son de cariz fundamentalmente económico.

Esto contribuye a que existieran retornos a partir de 1983, momento del inicio de la presidencia de Raúl Alfonsín. Otras personas, con otras características y otras razones, decidieron migrar y residir en España, como en otros países europeos. Las entradas y salidas de este colectivo producen un movimiento continuo desde entonces: los que llegan y los que, después de pocos o muchos años de residencia, retornan.

Este trabajo se basa en entrevistas en profundidad realizadas en Cataluña y en Buenos Aires, entre octubre de 1999 y marzo de 2000. Las primeras se refieren a personas que viven en Cataluña, de origen argentino al llegar, independientemente de la situación administrativa (sean españoles o estén en proceso de naturalización o tengan sólo la residencia) en el momento de la entrevista. Las realizadas en Buenos Aires abarcan a los argentinos que han vivido durante más de un año en algún país de la Comunidad Europea y han regresado. En todos los casos, se define como argentina a la persona nacida en Argentina, según el concepto jurídico del jus solis, vigente en ese país pero no en España.

Las respuestas de las personas que han aceptado realizar la entrevista han sido inusitadamente positivas respecto a la experiencia migratoria. Tienen algo que contar y mucho que compartir, no sólo respecto a la propia experiencia en algún país europeo, sino también consejos que dar y recomendaciones que hacer.

Para completar este panorama tan positivo, se hace necesario considerar las negativas a realizar las entrevistas. Si consideramos que las respuestas corresponden a un grupo homogéneo y particular, es menester estimar que las negativas también obedecen a ciertas razones, relacionadas con el mismo tema de la entrevista, más allá de las excusas por falta de tiempo o disponibilidad.

El objetivo de esta comunicación es perfilar la experiencia migratoria de un grupo de argentinos, tanto los que continúan residiendo en España como los que lo han hecho en varios países de la Unión Europea y han regresado. 
Esa experiencia se construye desde el final, un resultado positivo que se desea trasmitir, comunicar y compartir con los demás. Se reconoce que no representa al conjunto y que existen otras experiencias no aleccionadoras, por ejemplo, de las personas que no han querido responder.

La construcción del proceso migratorio en el discurso de cada uno de los entrevistados constituye una experiencia positiva, completa y pasada. Positiva, porque así lo declaran en términos explícitos. Completa, porque tanto si residen en España como si han vuelto a la Argentina, la decisión sobre el retorno ya ha sido tomada. Pasada, no sólo en términos individuales, sino también por irrepetible.

Existe una percepción generalizada de que el momento que se vive es en todo diferente y que las migraciones que se puedan iniciar ahora lo serán también. El contexto social en Europa ha cambiado y la sociedad argentina también lo ha hecho. Las experiencias que se relatan pertenecen al pasado, porque todo ha cambiado en los noventa: las personas que migran, los motivos, las expectativas, las sociedades de partida y de acogida, la respuesta de los estados.

La propuesta acerca de la construcción, individual y social, de la experiencia migratoria será desarrollada en tres partes. En la primera, se hará hincapié en las negativas a responder y en la diferenciación de dos grupos de migrantes. En la segunda y tercera, se analizarán las experiencias relatadas en las entrevistas y la construcción social de la experiencia migratoria. Por último, se considerará el contexto de estas respuestas positivas y las razones por las que este tipo de experiencia parece irrepetible.

\section{Las negativas a aceptar las entrevistas}

Para acceder a las personas que habían realizado la migración internacional no se ha recurrido a ningún fichero consular, ni a intermediación alguna de organismos oficiales. Los contactos directos y las recomendaciones mutuas (de unos entrevistados que proponían otros y de otras vías alternativas) permitieron acceder a esa red que se teje entre los migrantes residentes en un sitio y que enlaza unos con otros. En el caso de Buenos Aires, se realizaron múltiples tentativas de contactos, directas, a través de investigadores y de amigos.

Aún así, y a pesar de las presentaciones y de las mediaciones amistosas que se solicitaron, la cantidad de rechazos fue elevada. Resulta imprescindible considerar estas negativas, en la medida en que exige reconocer que las personas que responden no abarcan la totalidad de las experiencias posibles, sino aquéllas que se han elaborado y que se desean trasmitir, en la medida en que están, al menos, relativamente resueltas. La percepción de la migración como experiencia completa constituye la puerta de entrada a la entrevista. Ese acceso está cerrado en la medida en que se viva como un conflicto presente y las decisiones de retorno o la discusión de desagradables experiencias pasadas, todavía esté pendiente.

Las personas que se han negado lo hicieron porque no deseaban hablar de la cuestión. La negativa implica una visión de la migración y de la convivencia en países de la Unión Europea, que debería resultar absolutamente distinta de 
las relatadas y recogidas en las entrevistas que se analizan en este trabajo. Dado que habían sido contactadas a través de personas conocidas, las negativas se expresaron verbalmente, a través de llamadas telefónicas o de forma directa, a la persona amiga que solicitaba la entrevista. De ahí que se conozcan las razones expresadas y parte de las experiencias durante la migración.

Las negativas a hablar de la experiencia vivida obedecían a dos momentos diferentes en la vida del migrante. Por una parte, no se deseaba exponer una parte de la vida que aún no había sido resuelta. Por la otra, en el caso de las personas que ya habían retornado y la migración formaba parte del pasado, se negaron a hablar de una experiencia dura, insatisfactoria o desagradable. Hay temor en ambos casos. Miedo a reconocer que todavía no se ha tomado una decisión que aparece como pendiente. Insatisfacción por momentos vividos que no han podido elegirse y de una parte de la vida que se considera forzada, en la medida en que la migración, entre otras cuestiones, no fue libremente decidida.

Eso significa que los resultados hubieran sido otros, si todas las personas que se contactaron hubieran respondido. La migración se hubiera construido como una experiencia social con dos grupos antagónicos. Por una parte, el de personas satisfechas, que respondieron y que desean trasmitir un mensaje. Por el otro, el de aquéllos que no desean hablar de ello, porque todavía no han resuelto su situación o sólo desean olvidar lo sucedido.

Todas las personas entrevistadas, tanto en Cataluña como en Buenos Aires, habían realizado migraciones tempranas. No han respondido o no se ha accedido a aquella red de migraciones económicas de los años noventa, con otras motivaciones y con razones bien distintas. Con esto deseo poner de relieve que los profesionales exitosos o que han partido por razones meramente económicas, que se vinculan con los movimientos de la última década, no aparecen reflejados en el perfil que se construirá de los migrantes en este trabajo.

En los términos de los mismos entrevistados, el éxito no reside en el dinero, sino en el beneficio de una experiencia para el individuo, en lo que se puede trasmitir y en lo que otros pueden aprovechar, siguiendo sus consejos o teniendo en cuenta sus advertencias y en aquello que la sociedad puede ganar con los cambios. La experiencia ha sido exitosa y ello constituye, a mi entender, una buena razón para contarla.

Esto significa que las entrevistas abarcan un grupo reducido y preciso, cuyas características generales se pueden definir de la siguiente manera:

\section{Las migraciones han sido tempranas.}

2. No todas han tenido razones políticas, pero la situación general del país sí ha contribuido de manera directa a la toma de la decisión.

3. Rara vez se han realizado de manera individual o aislada, la mayoría de los entrevistados forman parte de una familia o de un grupo de parentesco que ha partido porque uno de ellos se ha visto obligado a hacerlo y los otros lo han seguido.

4. El nivel de instrucción es elevado, con formación universitaria, finalizada o inconclusa. 


\section{La construcción de la experiencia}

La mayoría de las personas que partieron temprano de Argentina, en la segunda mitad de los setenta, lo hicieron por razones políticas, personales o del familiar que acompañaban. Pocos de ellos, sin embargo, reclamaron el estatuto de refugiado político. Ninguno de los que respondieron lo hizo si vivían en un país del sur y oeste de Europa. Sólo los que residían en aquellos países cuyo Estado de bienestar reconocía sin mayores cortapisas a las personas en esa condición, como por ejemplo Suecia, solicitaron y obtuvieron el estatuto de refugiados.

Eso implica que aún cuando las personas no vivieron como refugiados en la mayoría de países de Europa, en el momento del retorno sí pudieron acceder, si así lo deseaban, a un estatuto particular que les permitió volver en condiciones especiales. Esos acuerdos fueron firmados entre el gobierno de Alfonsín y las Naciones Unidas, para facilitar el regreso de las personas que partieron involuntariamente de Argentina.

Las razones por las cuales no solicitaron el estatuto de refugiado en los países del sur fueron variadas, pero en general se puede decir que resultaba mucho más fácil optar a otra condición y obtener la regularización con el tiempo, que acceder a un estatuto que bien podría significar su marginación presente o futura.

En primer lugar, la España posfranquista no era terreno abonado, todavía, para declarar esa condición particular. La apreciación general se sustentó como argumento común en que hubiera sido contraproducente de cara a las autoridades. En segundo lugar, todos los que pudieron, buscaron los documentos necesarios para recuperar la nacionalidad de sus padres o abuelos, de origen europeo. Si bien viajaron con pasaporte argentino, resultaba mucho más fácil, incluso de cara al futuro, "recuperar» la nacionalidad del país donde se vivía que acreditar una condición que podía provocar rechazo. En tercer lugar, las migraciones a los países del sur todavía eran reducidas y el proceso de regularización no presentaba tantas dificultades.

Muchas de las personas residieron un tiempo sin papeles acreditativos, como turistas y sin permiso de trabajo. Su condición de profesionales, en muchos casos, ayudó a regularizar su situación. Ninguno de los que trabajaron sin permiso, se quejó de discriminación o de peores condiciones que los locales. Los profesionales fueron bien acogidos y las redes construidas por ellos permitieron no sólo la ayuda inicial a los que llegaban, sino también muestras de solidaridad constantes que contribuyeron a favorecer la inserción, sin mayores dificultades.

Hay quien prefirió vivir en situación irregular durante más de cuatro años. Eso no impide reconocer que se hubiera podido solicitar la residencia, al cumplir con todos los requisitos. Fue el hecho de sentir que estaba de paso y que la migración era sólo una etapa temporal y transitoria en su vida, lo que no brindaba motivos para hacer el trámite y cambiar su situación. Ello implica que carecía de motivos para regularizar su estancia, aún después de tanto tiempo. 
Es, además, el único caso no relacionado con ninguna razón política, ni personal ni de un familiar.

Cuando llegaron, ninguno estaba solo. A pesar de la situación y de que muchos salían de prisión con el billete de avión y con la única opción de partir al extranjero, familiares o amigos los esperaban en el primer aeropuerto de Europa, para acompañarlos, ayudarlos y no dejarlos solos en la primera parte de un periplo no buscado.

Los que tenían o podían conseguir alguna nacionalidad europea, fueron esperados por familiares, que les brindaron la bienvenida y quienes los alojaron en los primeros momentos. Aquéllos que viajaban junto con los familiares, porque no salían directamente de la cárcel, traían contactos de amigos o profesionales o conocidos que los alojaron y los introdujeron en el mundo laboral, de manera inmediata.

No se distinguen experiencias distintas por género. En ningún caso, las mujeres o los hombres se caracterizan por migraciones inducidas. Tantos unos como otros, se han encontrado en ambas situaciones. Las migraciones inducidas lo han sido, sobre todo, por razones de edad: los hijos de padres que han tenido que partir, pero no por el género.

Como los primeros contactos han sido indistintamente familiares o de amigos, tampoco se ha buscado formar un gueto o formar un grupo aparte de la sociedad local. Muy al contrario, todos han manifestado un deseo de integración y participación con los locales. A ello contribuyeron poderosamente las razones que tenían o la situación de la que gozaban. Por una parte, el hecho de tener hijos y de no querer que repitieran la experiencia del destierro, del dolor de partir, ha contribuido notablemente a la integración en la sociedad local. Por otra, en la medida en que familiares europeos los han recibido y los han tratado como de los suyos, no ha permitido ni discriminación, ni exclusión.

Las ventajas que presentaba el grupo de argentinos que realizaron una migración temprana fueron múltiples y las personas que respondieron a las entrevistas eran conscientes de ello. Resulta fundamental el hecho de que descendieran de europeos. Ello contribuyó de diversas maneras. En primer lugar, les permitió conseguir la nacionalidad o la residencia de manera casi inmediata. En segundo lugar, no se los distinguió, porque no se los identificaba como un grupo extraño. En tercer lugar, la situación social en origen, en todos los casos privilegiada, contribuyó a una inserción de clase que excluye la marginación. Si bien al llegar todos trabajaron en lo que pudieron, incluso en venta callejera; el nivel de instrucción era elevado y en poco tiempo consiguieron ocupaciones acordes con la titulación que poseían o una situación que pudo mejorar en corto tiempo. En cuarto lugar, las motivaciones personales, pero sobre todo familiares, contribuían a que se integraran, adoptaran los modos locales y no buscaran notoriedad como grupo o tuvieran reivindicaciones específicas que los distinguiera de la población local. Se puede decir que por cultura y etnia, por clase social y por deseo expreso, los argentinos que llegaron tempranamente a Europa se mimetizaron de tal manera que la población local los asimiló sin mayores inconvenientes. 
En este sentido, una de las negativas a realizar la entrevista partió de una pareja que había partido por razones políticas pero que no encontraron a nadie a quién recurrir al llegar a España. Las experiencias con trabajos temporales, mal pagados y una situación precaria, reforzaron un sentimiento de rechazo a la sociedad local y a su experiencia. En este caso, prima la no voluntariedad de la migración. Esta obligación de partir y residir fuera, a pesar de no quererlo, no fue resuelta de manera satisfactoria. En estos casos, los retornos son tempranos, en cuanto existió la posibilidad de hacerlo y el período de la migración, una parte negativa y rechazada de su vida, de la cual no desean ni hablar.

En cambio, según el propio reconocimiento de los entrevistados, si ha habido algún tipo de discriminación que los afectara, ésta ha sido positiva. Este tipo de trato desigual se centra en la Administración y no en las personas. Se reconoce que por el aspecto (en este caso se refiere no sólo al origen europeo, sino también a la clase social) han recibido un trato favorable. En primer lugar, las probabilidades de ser detenido y de que pidieran los documentos eran muy remotas. Cuando sucedía, caso excepcional incluso para la persona que vivió durante más de cuatro años sin regularizar su situación, no existió problema alguno al declararse turista. Se tenía el aspecto y el dinero suficiente para gozar de esa condición y se invocaba inequívocamente.

Cuando se deben solicitar los documentos para la regularización o se acude a la Administración, el trato implica una discriminación positiva, que no se busca, pero que sucede. Esperan los pobres. Los que tienen buen aspecto, por educación, por clase o por origen europeo, no tienen porqué hacerlo. Resultaba difícil negarse a este favoritismo de la Administración, argumentando que se desea ser como los otros. La realidad es que se es distinto y las autoridades lo reconocen.

Esa condición de persona de origen europeo, pero al mismo tiempo tercermundista favorece las relaciones con todos. Por una parte, con la sociedad local que no los rechaza como otros o distintos. Pero, al mismo tiempo, se goza de cierto prestigio con otros migrantes extranjeros, venidos de países del Tercer Mundo que encuentran un interlocutor bien visto por la Administración. Esto favoreció, en más de un caso, que las personas se dedicaran a defender y a interceder por comunidades de migrantes diferentes a las del país de origen al cual pertenecían.

De los casos relatados, se pueden describir dos situaciones. Por una parte, se encuentran los refugiados políticos en Suecia que necesitaban una persona que ya tuviera relaciones con la Administración, que supiera el idioma y que conociera los trámites o los vericuetos legales en las demandas precisas.

Por otra parte, estaban aquellas personas que, teniendo nacionalidad europea, se identificaban con otros extranjeros, extracomunitarios, a quienes ayudaron y por los cuales intercedieron ante la Administración. Gozaban, ante ellos, de una situación tan dual como privilegiada. Eran europeos y los locales los aceptaban como suyos. Habían nacido en el Tercer Mundo o en la periferia y se identificaban con los intereses de otros extranjeros, por lo que podían entenderlos, compartir puntos de vista y ser sus portavoces. 
Esta situación de dualidad y de pertenencia a la sociedad local, que da privilegios a otro mundo del que se viene y que se comparte con los demás migrantes, motivó respuestas unívocas frente a la pregunta sobre el racismo o sobre experiencias negativas. Todos, invariablemente, han respondido que no sufrieron experiencias racistas por parte de la Administración o de la sociedad local, en primera persona; pero que asistieron o conocen por terceras personas la experiencia que tienen que soportar las personas distintas, las que se ven diferentes porque lo son. Ésta es una razón más para reconocer que son un grupo particular y homogéneo, con puntos de contacto, pero también con grandes diferencias con otros migrantes extracomunitarios.

Evidentemente, los migrantes que han elaborado su experiencia son conscientes de la situación de privilegio de que gozaron, que no fue compartida, al menos por la mayoría de migrantes extraeuropeos. Las razones que ellos mismos adujeron fueron varias. Las de clase social y etnia figuran en primer lugar. Fueron conscientes que la primera distinción se realiza a través de la percepción de la diferencia y que ellos no eran distintos de los locales. Las razones políticas, como motivo de la migración en un determinado momento, que no se puede volver a invocar, figuran en segundo término. Una persona perseguida por razones políticas podía esperar un cierto reconocimiento de las otras personas de la sociedad, aunque no siempre de la Administración. Por eso, esa condición no se adujo oficialmente, aunque el reconocimiento del entorno sí podía producirse por esa razón.

Uno de los motivos para no iniciar migraciones en la actualidad, según se argumenta en las entrevistas, reside en que los motivos políticos tienen más prestigio que los económicos. Algunos migrantes consideran que las motivaciones de dinero, de ganar más, no pueden provocar la solidaridad que produjeron en su momento los exiliados argentinos y de otros países latinoamericanos en Europa.

De más está decir que, a pesar de la construcción de una experiencia positiva, los momentos desagradables fueron muchos. El desarraigo, el exilio, es difícil de asumir. Las depresiones o la desesperanza eran presa de muchos. Puede haber contribuido a ello el hecho de que la migración no había sido voluntaria. Este mismo hecho, al retorno o al decidir permanecer, también puede haber contribuido a la elaboración positiva de la experiencia y a la aceptación de ese período.

Esos mismos sentimientos negativos, compartidos por la comunidad de origen, pero sobre todo por los que partieron por los mismos motivos, también coadyuvó en la mayoría de los casos en la integración en la sociedad local, en vez de encerrarse con aquéllos en igual situación. La mayoría expresó que tenía amigos argentinos, pero frecuentaban a personas locales, en varios ámbitos. El consumo cultural era local, de productos locales. Podía haber encuentros entre argentinos, pero muchos huyeron de ellos para poder superar la sensación de vivir en un sitio y estar con la mente en otro, para no pensar constantemente en una sociedad a la que no se podía volver en esos momentos. 
No niego que existieran grupos cerrados que no se integraron en las sociedades locales y que permanecieron al margen. Pero, según declaración de los entrevistados, tanto en Cataluña como en Buenos Aires, esos grupos, a veces uno sólo, eran reducidos y conocidos por su rechazo a la comunidad local. Su deseo de no integración fue permanente. Luego de la posibilidad de retorno, ya no tuvieron razón de existencia. Las asociaciones de acogida también han desaparecido, puesto que los motivos solidarios que permitieron que se formaran han desaparecido al cambiar la situación en Argentina y al existir otros motivos para migrar.

\section{Migrar: una experiencia positiva, no siempre recomendable}

Cuando se ha interrogado a las personas sobre el juicio que merecía la etapa de la migración, todas salvo una contestó que positiva, a pesar del dolor y de la dureza de la experiencia; pero no todas la recomendarían. Existía una sola excepción a la construcción positiva de la experiencia, pero variados pareceres a la hora de considerar que esa experiencia podían llevarla a cabo otras personas o que en todo caso ellos la alentarían. Lo positivo en primera persona no siempre resultaba generalizable a experiencias futuras.

La única persona que contestó que la experiencia era negativa no definió su experiencia como migratoria, sino como un desplazamiento forzoso. Excluía, no sin motivo, del concepto de migración, la partida involuntaria, el vivir fuera y la imposibilidad temporal del retorno. Lo que puso en evidencia esta entrevista es que el retorno, que ahora es posible, no ha sido resuelto. La decisión sobre quedarse o partir todavía no había sido tomada. Mientras sea así, la experiencia no será completa y el juicio no es definitivo. Puede partir o puede permanecer; pero, en la medida en que se quede, la migración dejará de ser forzosa para convertirse en una experiencia volitiva.

Si bien las experiencias han sido elaboradas como algo positivo, esto es el resultado de una etapa acabada, sobre la cual se ha decidido, si no al principio al menos al final, de la cual se controla sobre todo el presente. Los que retornaron, como los que no lo hicieron, estiman que ese período ha sido positivo, en la medida en que les ha aportado muchas cosas que valoran, tanto en forma individual como en un discurso que desean trasmitir a los demás, en una palabra: comunicar.

Las razones por las cuales no recomendarían esta experiencia pueden ser tanto personales como sociales. En términos personales, se estima que la migración constituye una experiencia difícil que no cualquiera puede concluir con éxito. Algunos hablan de personas fuertes, independientes, no atadas a otros afectos, como requisito indispensable de la personalidad del migrante.

Sobre el contexto histórico, se reconoce que ha cambiado y ello puede desaconsejar las migraciones en la actualidad. Por una parte, el halo que podía conferir el hecho de no ser un migrante voluntario y de haber luchado por una opción democrática, se interpreta como una de las razones por las cuales la acogida en la sociedad local fue auspiciosa. Por el otro, el reconocimiento de que 
la situación en Europa se ha transformado, implica un giro negativo en la percepción de las sociedades europeas frente a los migrantes extranjeros.

Muchas personas, tanto en Cataluña como en Buenos Aires, reconocen que la percepción de la sociedad ahora es distinta: los brotes racistas y los conflictos de los últimos tiempos no recomiendan la migración. El hecho de que sólo se puedan argüir razones económicas, podría obstaculizar la acogida en la sociedad local. Existe una competencia por los recursos que antes no existía, había generosidad.

Los que han hecho alusión a esta situación perciben dos momentos diferentes. En el primero, los migrantes eran bien recibidos porque se recordaba, sobre todo en las sociedades europeas, que alguien de la familia o algún conocido había migrado antes, había partido a ese sitio, Argentina, cuando las condiciones no eran buenas, y había sido bien recibido. La primera etapa significa un retorno de los europeos (incluidos sus descendientes) a su país de origen. Al mismo tiempo que provocaba la reciprocidad; un deseo de acoger de manera generosa a aquéllos que lo habían hecho con sus familiares, no hace tanto tiempo.

La segunda etapa, la del creciente rechazo, que se vive en la actualidad, implica el trato a los que vienen como extraños; el olvido del propio pasado y de la experiencia migratoria de tantos familiares y la negación de ese tipo de situaciones que no hace tanto tiempo se han vivido en los círculos más cercanos, incluidos los familiares, o que hasta pudieran haber experimentado ellos mismos.

En esas circunstancias, la respuesta coherente de los entrevistados ha sido desear ser el otro, el europeo, desear que no se lo distinga; lo que es imposible y hace que la migración no sea recomendada. Querer ser el local significa que no se desea ninguna etiqueta como otro, diferente, frente a la imposibilidad de luchar contra sentimientos de rechazo y de exclusión. No se busca el reconocimiento, ni la integración. Se desea no sólo parecer local, sino también serlo, para evitar un rechazo creciente que de esta manera no los afectaría. Se niega el sí mismo. En cuanto no se puede ser otra cosa, no se recomienda la migración.

\section{La migración forma parte del pasado}

Tanto para los que regresaron como para aquéllos que no lo hicieron, la migración es una experiencia positiva en la medida en que forma parte del pasado. Representa una etapa en la vida que ha contribuido a que el presente sea mejor. En esa medida, existe un sentimiento compartido de trasmitir un mensaje, a veces de aviso, de advertencia o de simple recomendación.

La migración ha sido una experiencia que amplía los horizontes de aquéllos que la valoran. Les permite salir de una sociedad, la Argentina, que, aunque formada por contingentes de personas de distinto origen, no es tan distinta, ni tan diversa. Las sociedades europeas de la actualidad, pero también la Argentina actual y no la que conocieron los migrantes antes de la partida, resultan mucho más variadas en los orígenes continentales de las personas que ahora llegan. 
La Argentina, en la actualidad, incluso está conociendo reacciones adversas a una serie de comunidades extranjeras. Parece negar su propio pasado al no reconocer o no querer acordarse de las condiciones en las que llegaron muchos de sus padres o abuelos. Es un mentís de la historia. Ha habido migraciones por razones políticas o económicas. Los argentinos que vivieron en Europa o que todavía están, descendientes de los europeos que fueron, tuvieron que emprender, a su vez, un cierto camino de vuelta, porque la situación había cambiado.

Todos los que migraron tempranamente a Europa reconocieron que no podían vivir en esas condiciones y que buscaban las de antes, las que habían desaparecido. Por eso extraña que las sociedades que han obligado, forzadamente o no, a tantos miembros a partir, olviden tan pronto y den la espalda a los que llegan hoy.

La migración puede formar parte del pasado de las personas, en forma individual, pero forma parte del presente de todas las sociedades, porque es su historia, cercana y reciente, y la de sus miembros, muchas personas que todavía viven en un sitio diferente al que han nacido.

La migración resulta una experiencia completa y relativamente acabada en el caso explicado. Por una parte, se encuentra el retorno. Por el otro, está la permanencia, que se vuelve voluntaria. En términos estadísticos siempre se puede medir como migración la diferencia entre lugar de nacimiento y de residencia, por lo cual sólo serían migrantes aquéllos que permanecen en una sociedad diferente. Sin embargo, para aquéllos que retornaron tanto como para aquéllos que han decidido quedarse, la migración puede constituir una experiencia completa. En los términos temporales e inciertos en los que usualmente se mide, por una vez consideremos que existen experiencias acabadas.

\section{Bibliografía}

BonifazI, Corrado; Angela FerruZZA (1999). «Mujeres latinoamericanas en Italia: una nueva realidad del sistema de migraciones internacionales», Estudios Migratorios Latinoamericanos, Buenos Aires: CEMLA, 11.32, p. 169-177.

HernándEZ, José Lorenzo (1999). "The Nuyorican's Dilemma: Categorization of Returning Migrants in Puerto Rico», International Migration Review, Nueva York: Center for Migration Studies, 33, núm. 4, 128, p. 988-1013.

Massey, Douglas; Kristian EsPINOSA (1997). «What's Driving México U.S. Migration: A Theoretical, Empirical and Policy Analysis», American Journal of Sociology, USA, 102-4, p. 939-999.

LEIVA, María Luján (1997). Latinoamericanos en Suecia, una historia narrada por artistas y escritores, Uppsala University, Uppsala Multethnic Papers, 37.

- (1999). "Landscape in exile: Latin Americans in Sweden», Migration och mângfald, Uppsala: Centrum for Multietnisk Forskning, p. 301-320.

MORETTI, Enrico (1999). «Social Networks and Migrations: Italy 1876-1932», International Migratrion Review, Nueva York.

Rocha ReIS, Rosanna; Teresa Sales (comp.) (1999). Cenas do Brasil Migrante, Sao Paulo: Boitemp. 
Sanz Díaz, Benito; Josep Alenar Navarro; Concha Blat Mellado (eds.) (1999). Ciudadanía y Solidaridad en la Unión Europea, Valencia: Universidad de Valencia. SARRIBLE, Graciela (1998) «Sobre las migraciones comunitarias y extracomunitarias: contra la exclusión como calificativo genérico", Estudios Migratorios Latinoamericanos, Buenos Aires: CEMLA, 13-39, 239-256.

- (2000). «El regreso a Europa: argentinos en España», Scripta Nova: Revista Electrónica de Geografía y Ciencias Sociales, 59, Barcelona: Universidad de Barcelona.

- (2000). "Innovación social y migraciones: los argentinos como migrantes», comunicación al II Coloquio Internacional de Geocrítica: Innovación, Desarrollo y Medio Local: Dimensiones Sociales y Espaciales de la Innovación, Barcelona: Universidad de Barcelona.

SCHWARZSTEIN, Dora $(1997,12,37,423-444)$. «Actores sociales y política inmigratoria en la Argentina. La llegada de los republicanos españoles», Estudios Migratorios Latinoamericanos, Buenos Aires: CEMLA.

SUTCLIFFE, Bob (1998). Nacido en otra parte, Un ensayo sobre la migración internacional, el desarrollo y la equidad, Bilbao: Hegoa. 